\title{
Study of bioresources of salt lakes of Novosibirsk oblast
}

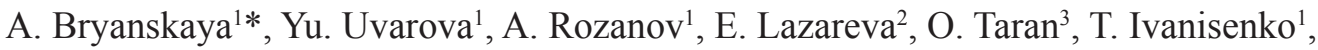 \\ S. Peltek ${ }^{1}$ \\ ${ }^{1}$ Institute of Cytology and Genetics SB RAS, Novosibirsk, Russia \\ ${ }^{2}$ Institute of Geology and Mineralogy SB RAS, Novosibirsk, Russia \\ ${ }^{3}$ Boreskov Institute of Catalysis SB RAS, Novosibirsk, Russia \\ *e-mail:alla@bionet.nsc.ru
}

Key words: methagenomic, biodiversity of microorganisms, salt lakes, microbial communities

Motivation and Aim: In the Kulunda and Baraba Steppes there are many brackish and saline lakes. These lakes are unstable water systems, characterized by significant fluctuations in the water level so the microorganism communities developing in them have various adaptations that allow them to withstand significant fluctuations in environmental factors. The aim of this study was to investigate the fundamentals of biogeochemistry and microbiology of extreme environments using small saline drainless lakes of the Novosibirsk oblast.

Methods and Algorithms: As a model object we took the Solenoye Lake (Novosibirsk oblast). We studied the composition of the benthic microbial community using parallel amplicon sequencing of the V3 variable region of the 16S rRNA gene, as well as geochemical parameters ( $\mathrm{pH}, \mathrm{Eh}$, cationic/anionic composition, gas components). We described and compared different layers of the bottom sediments and found relations between chemical composition and microbial content of the bottom sediments.

Results: As a result, it has been established that geochemical data on the study of the structure of the microbial community are well correlated. The upper $30-35 \mathrm{~cm}$ of the sediment by the ratio of the main groups of microorganisms are somewhat similar, and below, where the change occurs and the main geochemical and mineralogical indices of the substance, the composition of the community changes significantly. Throughout the sediment column, Actinobacteria and/or Proteobacteria predominated. In the layers of the columns the number of prokaryotic sequences found was from 96 to 414 . The proportion of archaeal sequences in the bottom sediments was 5.5-6.6\%, in water and microbial mathematics it was 2.0 and $1.2 \%$. In the lake water 50 prokaryotic sequences were identified, in the microbial mate -81 , in the columns of bottom sediments -390 . In water and microbial lake mate, Cyanobacteria and Proteobacteria dominated. It is established that the ecosystem of the Salt Lake is characterized by: (1) variations in geochemical parameters; (2) developing mass of microbial community with prevalence of cyanobacteria; (3) the presence of powerful layers of bottom sediments with a high taxonomic diversity of prokaryotes; (4) intensive development of the Artemia crab; (5) tracking changes in biological and geochemical characteristics in dynamics and extrapolating the data obtained to other lake ecosystems in the Novosibirsk region, characterized by a similar geochemical composition of natural solutions [1,2].

Acknowledgements: Supported by the RFBR (17-44-540815), by the Government of the Novosibirsk Region and by Budget Project of the IC\&G SB RAS (VI.58.1.8).

\section{References}

1. Lazareva E.V. et al. (2012) Research of elements distribution between components of salt lake system by SR'XRF technique. Poverkhnost $=$ J. Surface Investigation: X-Ray, Synchrotron and Neutron Techniques. 12:70-80. (in Russian)

2. Bryanskaya A.V. et al. (2016). BMC Microbiology. 16(1):S4.3. 